\title{
Differentiation between women with vulvovaginal symptoms who are positive or negative for Candida species by culture
}

\author{
Iara M. Linhares ${ }^{1,2}$, Steven S. Witkin ${ }^{2}$, Shirlei D. Miranda ${ }^{1}$, Angela M. Fonseca ${ }^{1}$, \\ Jose A. Pinotti ${ }^{1}$ and William J. Ledger ${ }^{2}$ \\ ${ }^{1}$ Department of Gynecology, Hospital das Clinicas, University of Sao Paulo, Sao Paulo, Brazil \\ ${ }^{2}$ Division of Immunology and Infectious Diseases, Department of Obstetrics and Gynecology, Weill Medical \\ College of Cornell University, New York, NY
}

Objective: To investigate whether clinical criteria could differentiate between women with vulvovaginitis who were culture positive or negative for vaginal Candida species.

Methods: Vulvovaginal specimens were obtained from $50 \mathrm{I}$ women with a vaginal discharge and/or pruritis. Clinical information and wet mount microscopy findings were obtained. All specimens were sent to a central laboratory for species identification.

Results: A positive culture for Candida species was obtained from 364 (72.7\%) of the specimens. C. albicans was identified in $86.4 \%$ of the positive cultures, followed by C. glabrata in $4.5 \%$, C. parapsilosis in $3.9 \%$, C. tropicalis in $2.7 \%$ and other Candida species in $1.4 \%$. Women with a positive Candida culture had an increased utilization of oral contraceptives ( $26.1 \%$ vs. $16.8 \%, p=0.02)$ and antibiotics $(8.2 \%$ vs. $0.7 \%, p=0.00 \mathrm{I})$, and were more likely to be pregnant $(9.1 \%$ vs. $3.6 \%, p=0.04)$ than the culture-negative women. Dyspareunia was more frequent in women without Candida ( $38.0 \%$ vs. $28.3 \%, p=0.03$ ) while vaginal erythema $(p=0.0 \mathrm{I})$ was more common in women with a positive Candida culture.

Conclusions: Although quantitative differences were observed, the presence of vaginal Candida vulvovaginitis cannot be definitively identified by clinical criteria.

Key words: Vulvovaginitis; CANDida SPecies; Differential Diagnosis

It is difficult to obtain accurate information regarding the prevalence and incidence of vulvovaginitis associated with a Candida species infection. Although up to $75 \%$ of women will acknowledge having had a vaginal Candida infection during their lifetime ${ }^{1}$, this diagnosis is suspect. The skyrocketing sales of over-the-counter medications for Candida vaginitis, at a rate many times that of the number of infected women, highlights the prevalent overdiagnosis of this disorder. Many women, and unfortunately also many clinicians, label any vaginal discharge, itching, pain or burning as a 'yeast' infection. In three studies, more than half of the women with a supposed vaginal yeast infection were misdiagnosed ${ }^{2-4}$.

Conversely, in many women a true vaginal Candida infection may remain unrecognized. Detection of a vaginal Candida infection by microscopic examination of a vaginal specimen diluted in potassium hydroxide is relatively insensitive, especially for non-albicans species ${ }^{3,5}$. False-positive microscopic examinations are also possible ${ }^{5}$ and are

Supported by Janssen-Cilag, Sao Paulo, Brazil

Correspondence to: Steven S. Witkin, Ph.D., Department of Obstetrics and Gynecology, Weill Medical College of Cornell University, 515 East 71st Street, New York, NY 10021, USA. Email: switkin@mail.med.cornell.edu 
probably more common than generally suspected. It is also possible to have Candida vulvovaginitis with a false-negative Candida culture. At least 3000 organisms/ml are necessary to obtain a positive culture $^{6}$.

In an attempt to more accurately characterize symptomatic women with a positive Candida species vaginal culture, and to differentiate them from women with vaginal symptoms due to other causes, a study was initiated in three cities in Brazil.

\section{MATERIALS AND METHODS}

This study was approved by the Clinical and Ethical Committee of Hospital das Clinicas, University of Sao Paulo, and informed written consent was obtained from all subjects. The study population consisted of 501 consecutive reproductive age women complaining of a vaginal discharge and/or vulvovaginal pruritis, seen as private patients in the Brazilian cities of Sao Paulo, Rio Grande de Sul and Salvador. Exclusion criteria included the use of immunosuppressive medications, vaginal medications or oral antifungal agents within the last 30 days.

Clinical and demographic data were collected at each center by a single participating physician. Signs and symptoms upon physical examination were standardized as much as possible between the different sites by providing common diagnostic criteria for erythema, edema, discharge and dysuria. Definitions were similar to those utilized by Eckert and colleagues ${ }^{7}$.

Specimens were obtained by scraping the vaginal walls with a cotton swab and immediately transferring the contents to a glass slide. A drop of saline was added and diagnosis of Candida was based on the observed presence of mycelium (branched hyphal elements) or blastospores (the unicellular yeast form). A second specimen was placed in transport medium and shipped to a central clinical laboratory. Specimens were cultured on Sabouraud agar containing chloramphenicol. Candida species were identified by the automated Amphotericin B (ATB) express method.

Comparisons between women with positive or negative Candida cultures for quantitative variables were analyzed by the Student's $t$-test for independent samples. The $\chi^{2}$ test was used to compare qualitative variables between both groups. Findings were considered significant at $p<0.05$.

\section{RESULTS}

Candida was detected by culture in 364 (72.7\%) of the subjects. The distribution of individual Candida species can be seen in Table 1. C. albicans was identified in $86.4 \%$ of the positive cultures, followed by C. glabrata (4.5\%), C. parapsilosis (3.9\%) and C. tropicalis $(2.7 \%)$. A presumed identification of Candida was made by microscopic examination in $87.1 \%$ of women with a positive culture and in $5.1 \%$ of those with a negative culture. Trichomonas vaginalis was present in $2.9 \%$ and $1.4 \%$ of women with a negative and positive Candida culture, respectively. Clue cells were observed in $16.8 \%$ of women with a negative culture and in $9.1 \%$ of women with a positive Candida culture $(p=0.01)$.

For all analyses, the patients were divided into two groups based on the presence or absence of a positive Candida culture. Demographics of women in both groups are shown in Table 2. A higher percentage of black women, but not of white women or those of other races, were present in the culture-negative group $(16.8 \%)$ than in the Candida culture-positive group (8.3\%) ( $p=0.01)$.

The relationship between predisposing factors and a positive or negative Candida culture is detailed in Table 3. Pregnancy $(9.1 \%$ vs. $3.6 \%$, $p=0.04)$, oral contraceptive usage $(26.1 \%$ vs. $16.8 \%, p=0.02)$ and current antibiotic use $(8.2 \%$ vs. $0.7 \%, p=0.001)$ were each associated with detection of a positive Candida culture. Conversely, a positive HIV serology $(7.3 \%$ vs. $3.3 \%$,

Table I Candida species identified by culture

\begin{tabular}{lc}
\hline Candida species & Percentage women positive \\
\hline albicans & 86.4 \\
glabrata & 4.5 \\
parapsilosis & 3.9 \\
tropicalis & 2.7 \\
krusei & 0.9 \\
guilliermondii & 0.6 \\
famata & 0.3 \\
pulcherrima & 0.3 \\
susitanii & 0.3 \\
\hline
\end{tabular}


Table 2 Demographics of women with vulvovaginitis and a positive or negative culture for Candida

\begin{tabular}{lcc}
\hline & $\begin{array}{c}\text { Candida } \\
(n=364)\end{array}$ & $\begin{array}{c}\text { No Candida } \\
(n=137)\end{array}$ \\
\hline Race & & \\
$\quad$ White & $79.0 \%$ & $73.7 \%$ \\
Black & $8.3 \%$ & $16.8 \% *$ \\
Oriental & $5.0 \%$ & $1.5 \%$ \\
Other & $7.7 \%$ & $8.0 \%$ \\
Age (years) & $32.2(10.1)^{\dagger}$ & $33.8(10.8)^{\dagger}$ \\
Sexually active & $93.1 \%$ & $84.7 \%$ \\
$>$ I Sexual partner & $7.5 \%$ & $3.1 \%$ \\
\hline
\end{tabular}

${ }^{*} p=0.01$ vs. black women with Candida; ${ }^{\dagger}$ standard deviation

Table 3 Predisposing factors associated with Candida species culture-positive and -negative vulvovaginitis

\begin{tabular}{lcc}
\hline & Candida & No Candida \\
\hline Diabetes & $2.8 \%$ & $4.4 \%$ \\
Pregnancy & $9.1 \% *$ & $3.6 \%$ \\
Oral contraception & $26.1 \% * *$ & $16.8 \%$ \\
IUD usage & $6.6 \%$ & $11.0 \%$ \\
Corticosteroid usage & $2.2 \%$ & $2.2 \%$ \\
Antibiotic usage & $8.2 \% * * *$ & $0.7 \%$ \\
HIV seropositive & $3.3 \%$ & $7.3 \%^{\dagger}$ \\
Prior STD & $9.7 \%$ & $17.5 \%{ }^{\dagger+}$ \\
\hline
\end{tabular}

${ }^{*} p=0.04,{ }^{*} p=0.02,{ }^{*} * *{ }^{*} p=0.001$ vs. women without Candida; ${ }^{+} p=0.05,{ }^{+\dagger} p=0.01$ vs. women with Candida

$p=0.05)$ and prior history of a sexually transmitted disease $(17.5 \%$ vs. $9.7 \%, p=0.01)$ were associated with a negative Candida culture. The HIV-seropositive women were at the earliest stages of their disease.

The relationship between patient-reported signs and symptoms and Candida culture findings is shown in Table 4. There was considerable overlap between the two groups in complaints of a vaginal discharge, vulvar pruritis and burning, dysuria and dyspareunia. Only dyspareunia was significantly different between the two groups: $38.0 \%$ in women with a negative culture vs. $28.3 \%$ in those with a positive Candida culture $(p=0.03)$.

Clinical findings in the patient groups are shown in Table 5. Although there was an overall high degree of similarity between subjects regardless of their Candida culture status, women with a positive culture had a higher prevalence of vaginal erythema $(p=0.01)$.
Table 4 Signs and symptoms of women with vulvovaginitis positive and negative for Candida by culture

\begin{tabular}{lcc}
\hline & Candida & No Candida \\
\hline Vulvar pruritis & $83.2 \%$ & $82.5 \%$ \\
Vulvar burning & $61.8 \%$ & $66.4 \%$ \\
Vaginal discharge & $85.7 \%$ & $87.6 \%$ \\
Dyspareunia & $28.3 \%$ & $38.0 \% *$ \\
Dysuria & $19.8 \%$ & $21.9 \%$ \\
$>$ I previous episode & $44.5 \%$ & $53.3 \%$ \\
Length of symptoms (days) & $18.6 \pm 8.6$ & $15.0 \pm 12.0$ \\
\hline
\end{tabular}

* $p=0.03$ vs. women with Candida

Table 5 Clinical findings in women with vulvovaginitis and positive or negative for Candida by culture

\begin{tabular}{lcc}
\hline & Candida & No Candida \\
\hline Vulvar edema & $10.7 \%$ & $9.5 \%$ \\
Vulvar erythema & $16.5 \%$ & $16.3 \%$ \\
Vaginal fissures & $18.4 \%$ & $16.1 \%$ \\
Vaginal erythema & $86.8 \% *$ & $59.2 \%$ \\
Leukorrhea & $84.9 \%$ & $88.6 \%$ \\
Excoriation & $9.9 \%$ & $8.8 \%$ \\
Cervical ectopy & $11.5 \%$ & $14.6 \%$ \\
Vesicles & $2.2 \%$ & $2.2 \%$ \\
Pustules & $2.2 \%$ & $2.2 \%$ \\
\hline
\end{tabular}

$*_{p}=0.01$ vs. women without Candida

\section{DISCUSSION}

Although some quantitative differences in the frequency of patient symptoms, clinical findings and predisposing factors were identified between groups of women who were culture-positive or culture-negative for Candida species, none of the evaluated criteria were pathognomonic for Candida in the vagina. Similar findings have been reported previously by others ${ }^{2,6-8}$. In a large study of women attending a sexually transmitted disease clinic, only $28 \%$ of 545 women with pruritis, burning or a vaginal discharge were C. albicansculture positive ${ }^{7}$. Thus, in symptomatic women, a positive wet mount or culture for Candida is necessary to assess whether this organism is present in the vagina.

The findings in the present study of associations between a positive Candida culture in symptomatic women and current oral contraceptive and antibiotic usage and pregnancy parallel earlier reports $^{9-11}$. We recognize that other studies have 
reported that women using oral contraceptives containing estrogen levels of $35 \mu \mathrm{g}$ or less did not have an increased rate of candida vulvovaginitis $^{7,11,12}$. This does not seem to be true, however, for our study population. The oral contraceptives used by the patients examined contained estrogen levels below $35 \mu \mathrm{g}$.

The etiology of the vaginal symptoms in the majority of our 137 Candida culture-negative patients remains undetermined. Bacterial vaginosis was a possible cause in 23 of these women, based on detection of clue cells by microscopy. An additional 4 women were positive for $T$. vaginalis by microscopic examination. Other possible causes of the observed symptoms, not evaluated in the present study, include allergic vaginitis, papillomavirus infection, desquamative vaginitis, cervicitis or estrogen deficiency. In addition, we acknowledge that in the Candida positive group, clinical signs and symptoms in at least some of the women may have been due to causes other than the presence of Candida species. However, the strong association between culture and wet mount suggests the presence of a high Candida concentration in the majority of the positive women and, therefore, an increased likelihood of Candidarelated symptomatology.

Host factors must also be taken into consideration when attempting to diagnose vulvovaginitis. It has been reported that there is no association between the Candida concentration in the vagina and clinical symptoms ${ }^{13}$. Women with Candida counts as low as 100 organisms/ml may be highly symptomatic while some women with vaginal Candida concentrations as high as 10 000/ml may be asymptomatic. Thus, women who are allergic to Candida antigens or products ${ }^{14}$ can become symptomatic even when vaginal concentrations of this organism are below the level detectable by culture. Furthermore, a low level of Candida in the vagina can synergize with histamine released in response to other allergens to induce prostaglandin $\mathrm{E}_{2}$ production ${ }^{15}$. This, in turn, inhibits the cellmediated immune response necessary to prevent Candida proliferation.

It is apparent that patient symptomatology, clinical examination and medical history are insufficient to distinguish vulvovaginitis associated with the presence of a Candida species from vulvovaginitis due to other causes. Initiation of antifungal treatment based solely on these criteria will be ineffective for the many women who are negative for this microorganism. In symptomatic women, a positive wet mount or culture for Candida species is necessary to determine whether this microbe is present.

\section{ACKNOWLEDGMENT}

We thank Luiz Carlos Severo for the Candida species identification.

\section{REFERENCES}

1. Hurley R, DeLouvois J. Candida vaginitis. Postgrad Med J 1979;55:645-7

2. Berg AO, Heidrich FE, Fihn SD, et al. Establishing the cause of symptoms in women in a family practice. J Am Med Assoc 1984;251:620-5

3. Nyirjesy P, Seeney SM, Grody MH, et al. Chronic fungal vaginitis: the value of cultures. Am J Obstet Gynecol 1995;173:820-3

4. Ledger WJ, Polaneczky MM, Yih MC, et al. Difficulties in the diagnosis of Candida vaginitis. Infect Dis Clin Pract 1999;9:66-9

5. Odds FC. Genital candidosis. Clin Exp Dermatol 1982;7:345-54

6. Schaaf VK, Perex-Stable EJ, Borchardt K. The limited value of symptoms and signs in the diagnosis of vaginal infections. Arch Intern Med 1990;150:1566-9

7. Eckert LO, Hawes SE, Stevens CE, et al. Vulvovaginal candidiasis: clinical manifestations, risk factors, management algorithm. Obstet Gynecol 1998;92:757-65

8. Sobel JD, Faro S, Force RW, et al. Vulvovaginal candidiasis: epidemiologic, diagnostic, and therapeutic considerations. Am J Obstet Gynecol 1998; 178:203-11

9. Spinillo A, Capuzzo F, Nicola S, et al. The impact of oral contraception on vulvovaginal candidiasis. Contraception 1995;51:293-7

10. Morton RS, Rashid S. Candidal vaginitis: natural history, predisposing factors and prevention. Proc $R$ Soc Med 1977;70(Suppl 4):3-6 
11. Ryley JF. Pathogenicity of Candida albicans with particular reference to the vagina. J Med Vet Mycology 1986;24:5-22

12. Gough PM, Warnock DW, Turner A, et al. Candidosis of the genital tract in non-pregnant women. Eur J Obstet Gynecol Reprod Biol 1985; 19:237-46

13. Meech RJ, Smith JM, Chew T. Pathogenic mechanisms in recurrent genital candidosis in women. N Z Med J 1985;771:1-5

RECEIVED 03/29/01; ACCEPTED 07/18/01
14. Witkin SS, Jeremias J, Ledger WJ. A localized vaginal allergic response in women with recurrent vaginitis. J Allergy Clin Immunol 1988;81:412-16

15. Witkin SS, Kalo-Klein A, Galland L, et al. Effect of Candida albicans plus histamine on prostaglandin E2 production by peripheral blood mononuclear cells from healthy women and women with recurrent candidal vaginitis. J Infect Dis 1991;164:396-9 


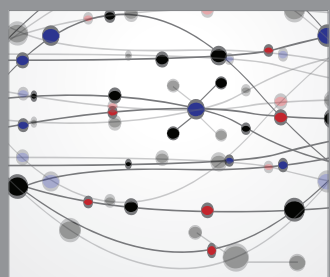

The Scientific World Journal
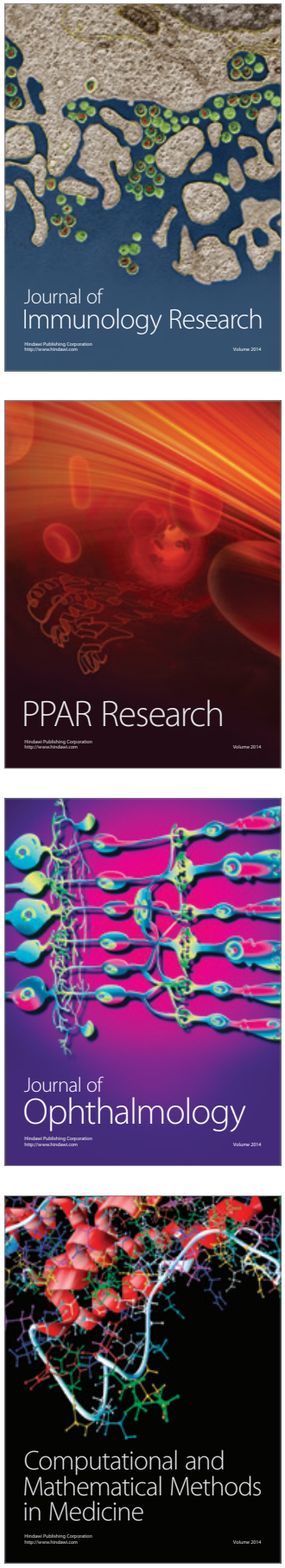

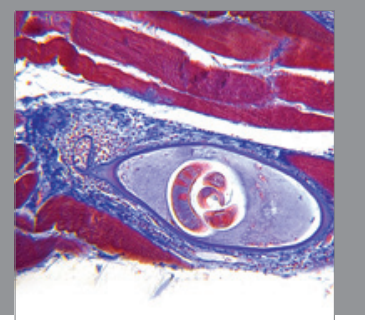

Gastroenterology

Research and Practice
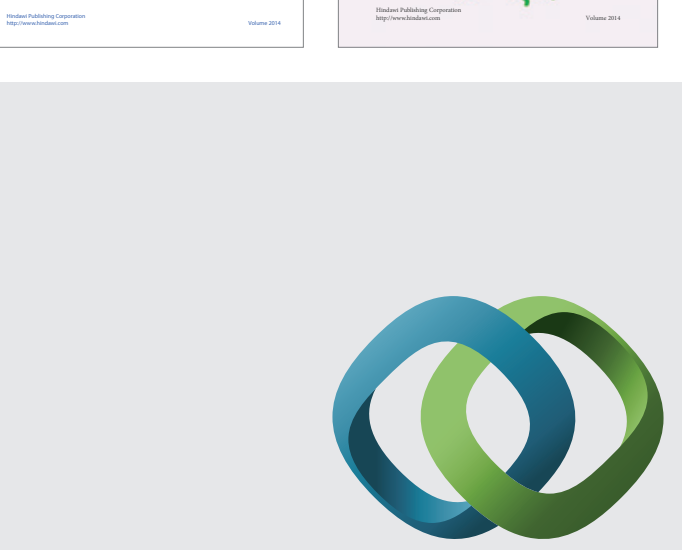

\section{Hindawi}

Submit your manuscripts at

http://www.hindawi.com
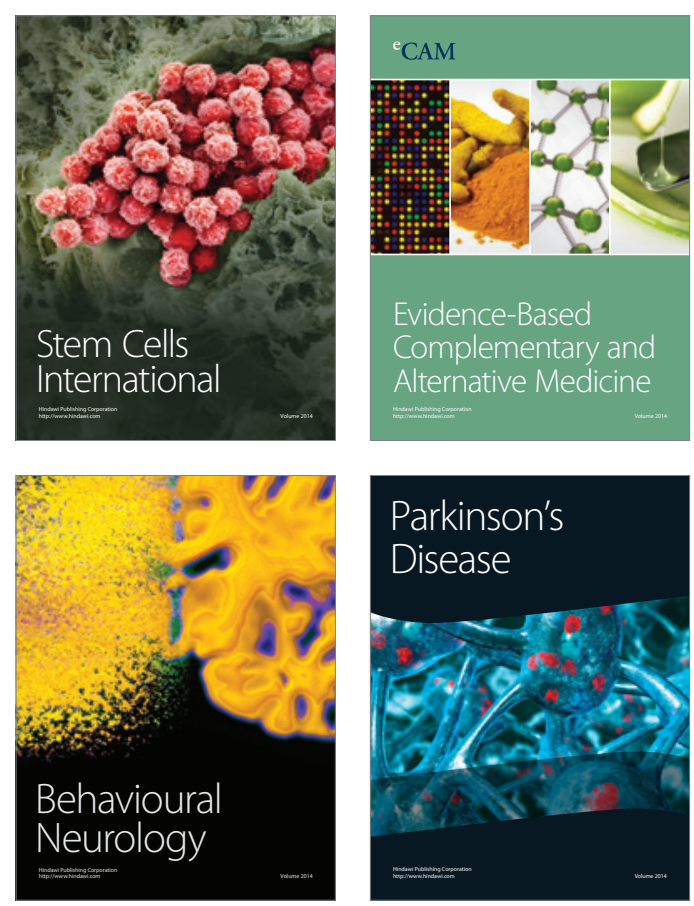

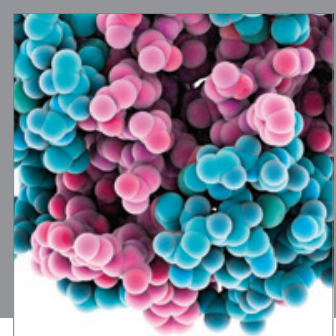

Journal of
Diabetes Research

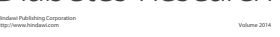

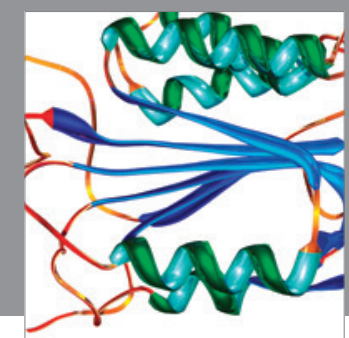

Disease Markers
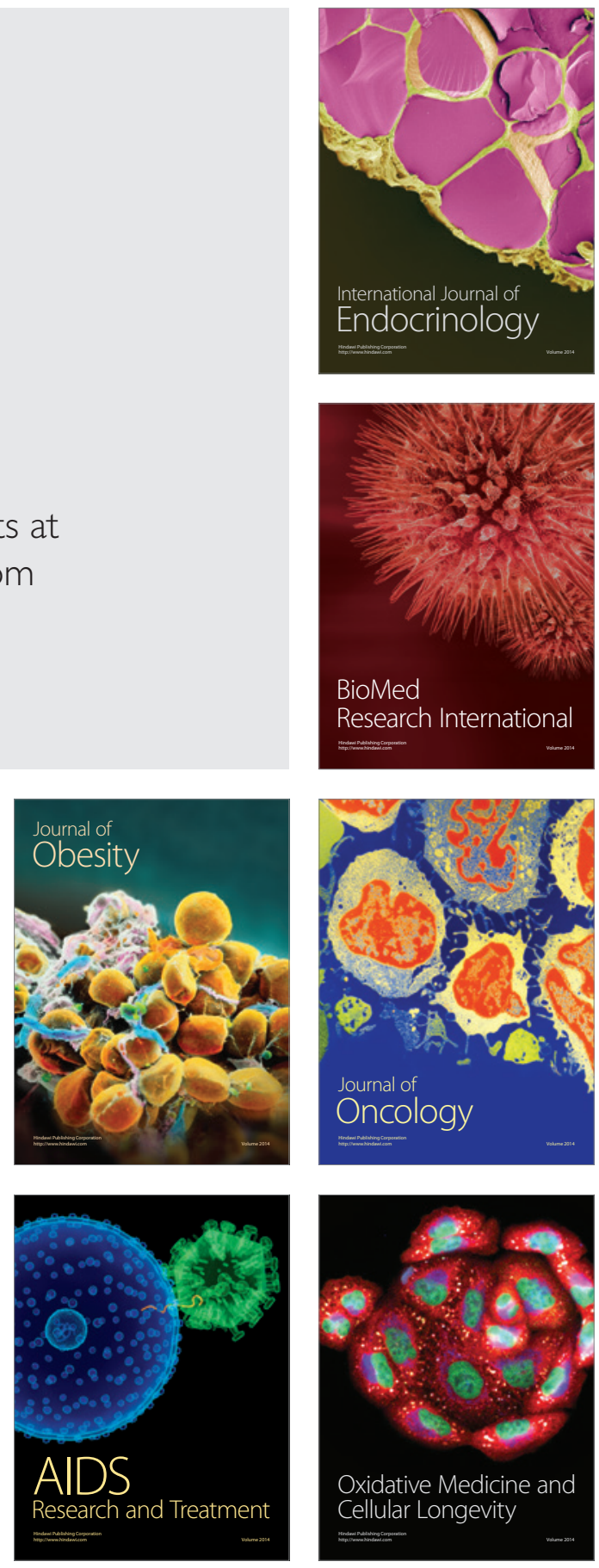